\title{
AN EXISTENCE RESULT \\ FOR A NONLINEAR BOUNDARY VALUE PROBLEM VIA TOPOLOGICAL ARGUMENTS
}

\author{
KHADIJAh SHARAF
}

\begin{abstract}
We investigate a nonlinear PDE related to the prescribing mean curvature problem on the boundary of the unit ball. We use variational and topological methods to prove the existence of at least one solution when the function to be prescribed satisfies at its critical points a non-degeneracy condition.
\end{abstract}

\section{Introduction and main results}

In this paper we consider the problem of existence of conformal scalar flat metric with prescribed boundary mean curvature on the unit $n$-dimensional ball. To be more specific, let $\mathbb{B}^{n}$ be the unit ball of $\mathbb{R}^{n}, n \geq 3$, with Euclidean metric $g_{0}$. Its boundary will be denoted by $\mathbb{S}^{n-1}$ and will be endowed with the standard metric still denoted by $g_{0}$. Let $H: \mathbb{S}^{n-1} \rightarrow \mathbb{R}$ be a given function, we study the problem of finding a conformal metric $g=u^{4 /(n-2)} g_{0}$ (here $u$ is a smooth positive function and the exponent $4 /(n-2)$ is used to make the next equation simpler) such that $R_{g}=0$ in $\mathbb{B}^{n}$ and $h_{g}=H$ on $\mathbb{S}^{n-1}$. Here $R_{g}$ is the scalar curvature associated to the metric $g$ in $\mathbb{B}^{n}$ and $h_{g}$ is the mean curvature of $g$

2010 Mathematics Subject Classification. Primary: 58E05, 35J65; Secondary: 53C21, 35B40.

Key words and phrases. Conformal metric; boundary mean curvature; lack of compactness; critical points at infinity; stable and unstable manifolds; retracts by deformation. 
on $\mathbb{S}^{n-1}$. This problem is equivalent to solving the following nonlinear boundary value equation:

$$
\begin{cases}\Delta u=0 & \text { in } \mathbb{B}^{n}, \\ \frac{\partial u}{\partial \nu}+\frac{n-2}{2} u=\frac{n-2}{2} H u^{n /(n-2)} & \text { on } \mathbb{S}^{n-1}\end{cases}
$$

where $\nu$ is the outward unit normal vector on $\mathbb{S}^{n-1}$ with respect to the metric $g_{0}$, see e.g. [5].

Equation (1.1) enjoys a variational structure. A natural space to look in for solutions is the Sobolev space $H^{1}\left(\mathbb{B}^{n}\right)$. Recall that by the regularity result of P. Cherrier [12], a weak solution of (1.1) is indeed a smooth solution. In equation (1.1), the exponent is $N-1$, where $N=2(n-1) /(n-2)$ is the critical case of the trace Soboev embedding $H^{1}\left(\mathbb{B}^{n}\right) \rightarrow L^{q}\left(\mathbb{S}^{n-1}\right)$. In virtue of the lack of compactness of this embedding, the Euler-Lagrange functional $J$ associated to our problem (see Section 2 for the definition of $J$ ) fails to satisfy the Palais-Smale condition; that is there exist non-compact sequences along which the functional is bounded and its gradient goes to zero. From the variational view point, it is the occurrence of critical points at infinity, that are the limit of the non-compact orbits of the gradient flow of $J$ (see Definition 2.1 below for more precision). This prevents the use of standard variational methods to prove existence of solutions. Moreover, besides the obvious necessary condition that $H$ should be positive somewhere, there is at least another obstruction to solving the problem, the so-called Kazdan-Warner condition [20].

Problem (1.1) has been studied by Escobar and Garcia [16] in dimension 3, who proved that blow-ups of solutions of subcritical approximations occur at one point and gave an index-count formula reminiscent to the one given by Bahri and Coron [8] and Chang, Gursky and Yang [10] for the prescribed scalar curvature on three dimensional sphere. We point out that the index formula of [16] has an equivalent in dimension 4, see [13] and [2]. However, the method cannot be generalized to higher dimension $n \geq 5$ under the non-degeneracy condition, since the corresponding index-count criteria, when taking into account all critical points at infinity is always equal to 1 . There have been many works devoted to the existence results, trying to understand under what condition (1.1) is solvable. For details see [1]-[4], [11], [13]-[19], [22] and the references therein.

Motivated by the work of [1] and [4], and aiming to include a larger class of functions $H$ in the existence results for (1.1), we develop in this paper a topological approach which enables us to provide sufficient conditions on $H$ weaker than those of [2], [3], [11], [13], [16] to obtain solution of (1.1) for every dimension $n \geq 3$. Our method hinges on the theory of critical points at infinity of A. Bahri [6]. 
To state our existence result, we need to introduce the assumptions that we will use and some known facts. Throughout this paper, we assume that $H: \mathbb{S}^{n-1} \rightarrow \mathbb{R}, n \geq 3$, is a positive Morse function satisfying the following nondegeneracy condition:

$$
\Delta H(y) \neq 0 \quad \text { whenever } \nabla H(y)=0 .
$$

We denote $\mathcal{K}^{+}:=\left\{y \in \mathbb{S}^{n-1}, \nabla H(y)=0\right.$ and $\left.-\Delta H(y)>0\right\}$. To every tuple of distinct elements $y_{i_{1}}, \ldots, y_{i_{p}}$ of $\mathcal{K}^{+}$, we associate the matrix $M\left(y_{i_{1}}, \ldots, y_{i_{p}}\right)=$ $\left(M_{j k}\right)$ defined by

$$
\begin{cases}M_{j j}=-c \frac{\Delta H\left(y_{i_{j}}\right)}{\left(H\left(y_{i_{j}}\right)\right)^{n-1}} & \text { for } j=1, \ldots, p, \\ M_{j k}=-c^{\prime} \frac{G\left(y_{i_{j}}, y_{i_{k}}\right)}{\left(H\left(y_{i_{j}}\right) H\left(y_{i_{k}}\right)\right)^{(n-2)} / 2} & \text { for } j \neq k,\end{cases}
$$

where

$$
c=\int_{\mathbb{R}^{n-1}} \frac{|z|^{2}}{\left(1+|z|^{2}\right)^{n-1} d z}, \quad c^{\prime}=\int_{\mathbb{R}^{n-1}} \frac{d z}{\left(1+|z|^{2}\right)^{n-2}},
$$

and $G$ denotes the Green's function related to problem (1.1).

Let $\rho\left(y_{i_{1}}, \ldots, y_{i_{p}}\right)$ be the least eigenvalue of $M\left(y_{i_{1}}, \ldots, y_{i_{p}}\right)$. It was first pointed out by A. Bahri [6], that when the self interactions of functions failing the Palais-Smale condition and the mutual interactions between two such different functions are of the same size, the function $\rho$ plays a fundamental role in the existence of solutions to problems like (1.1). Regarding problem (1.1), such kind of phenomenon appears in dimension 4, see [2]. However in dimension $n \geq 5$, the interaction of two different functions failing the Palais-Smale condition is negligible with respect to the self interactions, while in dimension 3 the reverse happens. When it comes to the 4 -dimensional case, we will assume that $\rho\left(y_{i_{1}}, \ldots, y_{i_{p}}\right) \neq 0$, for all $y_{i_{1}}, \ldots, y_{i_{p}} \in \mathcal{K}^{+}$.

Let $\mathcal{C}^{+}$be the following set:

$$
\begin{gathered}
\mathcal{C}^{+}:=\left\{\left(y_{i_{1}}, \ldots, y_{i_{p}}\right) \in\left(\mathcal{K}^{+}\right)^{p}, p \geq 1, \text { with } y_{i_{j}} \neq y_{i_{k}}, \text { for all } j \neq k\right\}, \quad \text { if } n \geq 5, \\
\mathcal{C}^{+}:=\left\{\left(y_{i_{1}}, \ldots, y_{i_{p}}\right) \in\left(\mathcal{K}^{+}\right)^{p}, p \geq 1, \text { with } y_{i_{j}} \neq y_{i_{k}},\right. \\
\left.\quad \text { for all } j \neq k \text { and } \rho\left(y_{i_{1}}, \ldots, y_{i_{p}}\right)>0\right\}, \quad \text { if } n=4,
\end{gathered}
$$

and $\mathcal{C}^{+}:=\mathcal{K}^{+}$, if $n=3$. The following known result describes the lack of compactness property associated to problem (1.1).

Proposition 1.1. Assume that (1.1) has no solution on $\mathbb{B}^{n}, n \geq 3$. Then the critical points at infinity of the associated variational problem correspond to:

$$
\left(y_{i_{1}}, \ldots, y_{i_{p}}\right)_{\infty}:=\sum_{j=1}^{p} \frac{1}{\left(H\left(y_{i_{j}}\right)\right)^{(n-2) / 2}} \delta_{\left(y_{i_{j}}, \infty\right)},
$$


where $\left(y_{i_{1}}, \ldots, y_{i_{p}}\right) \in \mathcal{C}^{+}$. The Morse index of such critical point at infinity is equal to

$$
i\left(y_{i_{1}}, \ldots, y_{i_{p}}\right)_{\infty}:=p-1-\sum_{j=1}^{p}\left(n-1-\operatorname{ind}\left(H, y_{i_{j}}\right)\right),
$$

here $\operatorname{ind}(H, y)$ denotes the Morse index of $H$ at $y$ and $\delta_{(a, \lambda)}, a \in \mathbb{S}^{n-1}$ and $\lambda>0$, are the solutions of the Yamabe type problem when $H$ equals to a constant.

The above result was proved in [3, Proposition 5.5] for the dimension 3 , in [2, Corollary 6.3] for the dimension 4 and in [3, Proposition 4.2] for the dimension $n \geq 5$.

Let $\mathcal{C}^{\infty}$ be the set of critical points at infinity given by

$$
\mathcal{C}^{\infty}=\left\{w_{p}^{\infty}:=\left(y_{i_{1}}, \ldots, y_{i_{p}}\right)_{\infty},\left(y_{i_{1}}, \ldots, y_{i_{p}}\right) \in \mathcal{C}^{+}\right\} .
$$

If $w_{p}^{\infty} \in \mathcal{C}^{\infty}$, let $W_{u}^{\infty}\left(w_{p}^{\infty}\right)$ designates its unstable manifold and $W_{s}^{\infty}\left(w_{p}^{\infty}\right)$ its stable manifold relative to the $C^{1}$-vector field $(-\partial J)$, the opposite of the gradient vector field for $J$.

Let $k_{0} \in \mathbb{N}$ and let $S_{k_{0}}^{\infty}$ be a subset of $\mathcal{C}_{k_{0}}^{\infty}$, where $\mathcal{C}_{k_{0}}^{\infty}:=\left\{w_{p}^{\infty} \in \mathcal{C}^{\infty}, i\left(w_{p}^{\infty}\right)\right.$ $\left.\leq k_{0}\right\}$. We consider the following set:

$$
W_{u}\left(S_{k_{0}}^{\infty}\right):=\bigcup_{w_{p}^{\infty} \in S_{k_{0}}^{\infty}} W_{u}^{\infty}\left(w_{p}^{\infty}\right) .
$$

Observe that $W_{u}\left(S_{k_{0}}^{\infty}\right)$ is contractible in $\Sigma^{+}$, the space of variation of $J$, since $\Sigma^{+}$is a contractible space. Therefore, $W_{u}\left(S_{k_{0}}^{\infty}\right)$ admits at least one contraction in $\Sigma^{+}$.

$\left(\mathrm{A}_{1}\right)$ Assume that $W_{u}\left(S_{k_{0}}^{\infty}\right)$ possesses a contraction $\Theta_{0}\left(W_{u}\left(S_{k_{0}}^{\infty}\right)\right)$ in $\Sigma^{+}$with the following property:

$$
\Theta_{0}\left(W_{u}\left(S_{k_{0}}^{\infty}\right)\right) \cap W_{s}^{\infty}\left(w_{p}^{\infty}\right)=\emptyset, \quad \text { for all } w_{p}^{\infty} \in \mathcal{C}_{k_{0}+1}^{\infty} \backslash S_{k_{0}}^{\infty} .
$$

Here $\mathcal{C}_{k_{0}+1}^{\infty}:=\left\{w_{p}^{\infty} \in \mathcal{C}^{\infty}, i\left(w_{p}^{\infty}\right) \leq k_{0}+1\right\}$

THEOREM 1.2. Under assumption $\left(\mathrm{A}_{1}\right)$, if

$\left(\mathrm{A}_{2}\right) \sum_{w_{p}^{\infty} \in S_{k_{0}}^{\infty}}(-1)^{i\left(w_{p}^{\infty}\right)} \neq 1$,

then (1.1) has a solution.

We will show that many interesting existence results are applications of Theorem 1.2, particulary those of [2], [3], [11], [13], [16]. In Section 3, we will consider some situations where the aforesaid results do not provide a solution to problem (1.1), but by Theorem 1.2 we derive that (1.1) admits a solution. More precisely, we shall prove that Theorem 1.2 is a proper generalization of the following results. 
Corollary 1.3. In the 3- and 4-dimensional cases, if

$$
\sum_{w_{p}^{\infty} \in \mathcal{C}^{\infty}}(-1)^{i\left(w_{p}^{\infty}\right)} \neq 1
$$

then (1.1) has a solution.

The result of Corollary 1.3 was proved in [3] and [16] in the 3-dimensional case and in [2] and [13] in the 4-dimensional case. The used method in [13] and [16] is based on a fine blow-up analysis of some subcritical approximations. While the approach of [2] and [3] consists of studying the critical points at infinity of the associated variational problem.

Corollary 1.4. Assume that $H$ is of the form $H=1+\varepsilon H_{0}, H_{0} \in C^{2}\left(\mathbb{S}^{n-1}\right)$ and $|\varepsilon|$ is small, then problem (1.1) has a solution provided

$$
\sum_{y \in \mathcal{K}^{+}}(-1)^{n-1-\operatorname{ind}(H, y)} \neq 1 .
$$

The result of Corollary 1.4 was proved in [11], see also [4].

In Section 2, we set up the variational structure of problem (1.1) and we give some auxiliary tools. In Section 3, we provide the proof of Theorem 1.2 and discuss the relation between this theorem and its corollaries.

\section{Variational structure}

In this section, we recall the functional setting and the variational problem and its main features. Problem (1.1) has a variational structure. The EulerLagrange functional is

$$
J(u)=\left(\int_{\mathbb{S}^{n-1}} H u^{2(n-1) /(n-2)} d \sigma_{g_{0}}\right)^{(2-n) /(n-1)},
$$

defined on $H^{1}\left(\mathbb{B}^{n}\right)$ equipped with the norm

$$
\|u\|^{2}=\int_{\mathbb{B}^{n}}|\nabla u|^{2} d v_{g_{0}}+\frac{n-2}{2} \int_{\mathbb{S}^{n-1}} u^{2} d \sigma_{g_{0}},
$$

where $d v_{g_{0}}$ and $d \sigma_{g_{0}}$ denote the Riemannian measure on $\mathbb{B}^{n}$ and $\mathbb{S}^{n-1}$ induced by the metric $g_{0}$. We denote by $\Sigma$ the unit sphere of $H^{1}\left(\mathbb{B}^{n}\right)$ and we set $\Sigma^{+}=\{u \in \Sigma, u \geq 0\}$. The exponent $2(n-1) /(n-2)$ is critical for the Sobolev trace embedding $H^{1}\left(\mathbb{B}^{n}\right) \rightarrow L^{q}\left(\mathbb{S}^{n-1}\right)$. As this embedding is not compact, the functional $J$ does not satisfy the Palais-Smale condition.

In order to characterize the sequences failing the Palais-Smale condition, we need to introduce some notations. We will use the notation $x$ for the variables belonging to the unit ball $\mathbb{B}^{n}$ or to the half space $\mathbb{R}_{+}^{n}$ defined by $\mathbb{R}_{+}^{n}:=\{x \in$ $\left.\mathbb{R}^{n}, x_{n}>0\right\}$. We will also use the notation $x=\left(x^{\prime}, x_{n}\right)$ for $x \in \mathbb{R}_{+}^{n}$. It will be convenient to perform some stereographic projection in order to reduce the above 
problem to $\mathbb{R}_{+}^{n}$. Let $D^{1,2}\left(\mathbb{R}_{+}^{n}\right)$ denote the completion of $C_{c}^{\infty}\left(\overline{\mathbb{R}_{+}^{n}}\right)$, with respect to the Dirichlet norm. The stereographic projection $\pi_{q}$ through an appropriate point $q \in \mathbb{S}^{n-1}$ induces an isometry $i: H^{1}\left(\mathbb{B}^{n}\right) \rightarrow D^{1,2}\left(\mathbb{R}_{+}^{n}\right)$ according to the following formula:

$$
i u(x)=\left(\frac{2}{\left|x^{\prime}\right|^{2}+\left(x_{n}+1\right)^{2}}\right)^{(n-2) / 2} u\left(\frac{2 x^{\prime}}{\left|x^{\prime}\right|^{2}+\left(x_{n}+1\right)^{2}}, \frac{\left|x^{\prime}\right|^{2}+x_{n}-1}{\left|x^{\prime}\right|^{2}+\left(x_{n}+1\right)^{2}}\right),
$$

where $x^{\prime}=\left(x_{1}, \ldots, x_{n-1}\right)$. For $a \in \partial \mathbb{R}_{+}^{n}$ and $\lambda>0$, we define the function

$$
\widetilde{\delta}_{(a, \lambda)}(x)=c_{0} \frac{\lambda^{(n-2) / 2}}{\left(\left(1+\lambda x_{n}\right)^{2}+\lambda^{2}\left|x^{\prime}-a^{\prime}\right|^{2}\right)^{(n-2) / 2}},
$$

where $x \in \mathbb{R}_{+}^{n}$, and $\bar{c}$ is chosen such that $\widetilde{\delta}_{a, \lambda}$ satisfies the following equation:

$$
\left\{\begin{aligned}
\Delta u & =0 \quad \text { and } \quad u>0 & & \text { in } \mathbb{R}_{+}^{n} \\
-\frac{\partial u}{\partial x_{n}} & =u^{n /(n-2)} & & \text { on } \partial \mathbb{R}_{+}^{n} .
\end{aligned}\right.
$$

Set

$$
\delta_{a, \lambda}=i^{-1}\left(\widetilde{\delta}_{(a, \lambda)}\right) .
$$

For $\varepsilon>0, p \in \mathbb{N}^{*}$, let us define

$$
V(p, \varepsilon)= \begin{cases}u \in \Sigma \text { s.t. } \exists a_{1}, \ldots, a_{p} \in \mathbb{S}^{n-1}, \exists \alpha_{1}, \ldots, \alpha_{p}>0, & \\ \exists \lambda_{1}, \ldots, \lambda_{p}>\varepsilon^{-1} \text { with }\left\|u-\sum_{i=1}^{p} \alpha_{i} \delta_{\left(a_{i}, \lambda_{i}\right)}\right\|<\varepsilon, \quad \varepsilon_{i j}<\varepsilon \forall i \neq j, \\ \text { and }\left|\frac{\alpha_{i}^{2 /(n-2)} H\left(a_{i}\right)}{\alpha_{j}^{2 /(n-2)} H\left(a_{j}\right)}-1\right|<\varepsilon & \forall i, j=1, \ldots, p,\end{cases}
$$

where

$$
\varepsilon_{i j}=\left(\frac{\lambda_{i}}{\lambda_{j}}+\frac{\lambda_{j}}{\lambda_{i}}+\lambda_{i} \lambda_{j}\left|a_{i}-a_{j}\right|^{2}\right)^{(2-n) / 2} .
$$

If $u$ is a function in $V(p, \varepsilon)$, one can find an optimal representation following [7]. Namely we have

Proposition 2.1. For any $p \in \mathbb{N}^{*}$, there is $\varepsilon_{p}>0$ such that if $\varepsilon \leq \varepsilon_{p}$ and $u \in V(p, \varepsilon)$, then the following minimization problem:

$$
\min _{\alpha_{i}>0, \lambda_{i}>0, a_{i} \in S^{n-1}}\left\|u-\sum_{i=1}^{p} \alpha_{i} \delta_{\left(a_{i}, \lambda_{i}\right)}\right\|,
$$

has a unique solution $(\alpha, \lambda, a)$, up to a permutation.

In particular, we can write $u$ as follows:

$$
u=\sum_{i=1}^{p} \alpha_{i} \delta_{\left(a_{i}, \lambda_{i}\right)}+v,
$$

where $v$ belongs to $H^{1}\left(\mathbb{B}^{n}\right)$ and it satisfies the condition 
$\left(\mathrm{V}_{0}\right)\langle v, \psi\rangle=0$ for $\psi \in\left\{\delta_{i}, \frac{\partial \delta_{i}}{\partial \lambda_{i}}, \frac{\partial \delta_{i}}{\partial a_{i}}, i=1, \ldots, p\right\}$,

here, $\delta_{i}=\delta_{\left(a_{i}, \lambda_{i}\right)}$ and $\langle\cdot, \cdot\rangle$ denotes the scalar product defined on $H^{1}\left(\mathbb{B}^{n}\right)$ by

$$
\langle u, v\rangle=\int_{\mathbb{B}^{n}} \nabla u \nabla v d v_{g_{0}}+\frac{n-2}{2} \int_{\mathbb{S}^{n-1}} u v d \sigma_{g_{0}} .
$$

The failure of the Palais-Smale condition can be characterized as follows, see $[3]$ and $[21]$.

Proposition 2.2. Let $\left(u_{k}\right)$ be a sequence in $\Sigma^{+}$such that $J\left(u_{k}\right)$ is bounded and $\partial J\left(u_{k}\right)$ goes to zero. Then there exist an integer $p \in \mathbb{N}^{*}$, a sequence $\left(\varepsilon_{K}\right)>$ 0 , $\varepsilon_{k}$ tends to zero, and an extracted subsequence of $u_{k}$ 's, again denoted $\left(u_{k}\right)$, such that $u_{k} \in V\left(p, \varepsilon_{k}\right)$.

Next, we give a definition extracted from [7].

Definition 2.3. A critical point at infinity of $J$ on $\Sigma^{+}$is a limit of a flow line $u(s)$ of the equation

$$
\left\{\begin{array}{l}
\frac{\partial u}{\partial s}=-\partial J(u(s)), \\
u(0)=u_{0},
\end{array}\right.
$$

such that $u(s)$ remains in $V(p, \varepsilon(s))$ for $s \geq s_{0}$. Here $\varepsilon(s)$ is some positive function tending to zero when $s \rightarrow+\infty$. Using Proposition 2.1, $u(s)$ can be written as

$$
u(s)=\sum_{i=1}^{p} \alpha_{i}(s) \delta_{\left(a_{i}(s), \lambda_{i}(s)\right)}+v(s) .
$$

Denoting $\widetilde{\alpha}_{i}:=\lim _{s \rightarrow+\infty} \alpha_{i}(s), \widetilde{y}_{i}:=\lim _{s \rightarrow+\infty} a_{i}(s)$, we denote by

$$
\sum_{i=1}^{p} \widetilde{\alpha}_{i} \delta_{\left(\widetilde{y}_{i}, \infty\right)} \text { or } \quad\left(\widetilde{y}_{1}, \ldots, \widetilde{y}_{p}\right)_{\infty}
$$

such a critical point at infinity.

\section{Proof of existence results}

This section is devoted to the proof of Theorem 1.2 and its corollaries. We will discuss how Theorem 1.2 is considered as a real generalization of Corollaries 1.3 and 1.4.

Proof of Theorem 1.2. We argue by contradiction, we suppose that problem (1.1) has no solution. It follows from Proposition 1.1 that the only critical points at infinity of $J$ are the elements of $\mathcal{C}^{\infty}$ defined in (1.2). 
For $k_{0} \in \mathbb{N}$ and for $S_{k_{0}}^{\infty}$ a subset of $\mathcal{C}_{k_{0}}^{\infty}:=\left\{w_{p}^{\infty} \in \mathcal{C}^{\infty}, i\left(w_{p}^{\infty}\right) \leq k_{0}\right\}$, we define

$$
W_{u}\left(S_{k_{0}}^{\infty}\right)=\bigcup_{w_{p}^{\infty} \in S_{k_{0}}^{\infty}} W_{u}^{\infty}\left(w_{p}^{\infty}\right),
$$

where $W_{u}^{\infty}\left(w_{p}^{\infty}\right)$ is the unstable manifold of $w_{p}^{\infty}$ and $i\left(w_{p}^{\infty}\right)$ is its Morse index. Observe that $\Sigma^{+}$is a contractible set, therefore there exists at least one contraction

$$
\Theta:[0,1] \times W_{u}\left(S_{k_{0}}^{\infty}\right) \rightarrow \Sigma^{+}, \quad(t, u) \mapsto \Theta(t, u),
$$

$\Theta(0, u)=u$, for all $u \in W_{u}\left(S_{k_{0}}^{\infty}\right), \Theta$ is continuous and $\Theta(1, u)=u_{0}$, a fixed element of $\Sigma^{+}$. Let

$$
\Theta\left(W_{u}\left(S_{k_{0}}^{\infty}\right)\right):=\Theta\left([0,1] \times W_{u}\left(S_{k_{0}}^{\infty}\right)\right) .
$$

Deform $\Theta\left(W_{u}\left(S_{k_{0}}^{\infty}\right)\right)$ by using the gradient flow-lines of $(-\partial J)$. We say that a critical point at infinity $w_{p}^{\infty}$ is dominated by $\Theta\left(W_{u}\left(S_{k_{0}}^{\infty}\right)\right)$ and denote $w_{p}^{\infty}<$ $\Theta\left(W_{u}\left(S_{k_{0}}^{\infty}\right)\right)$ if

$$
\Theta\left(W_{u}\left(S_{k_{0}}^{\infty}\right)\right) \cap W_{s}\left(w_{p}^{\infty}\right) \neq \emptyset
$$

By using a deformation lemma, see Proposition 7.24 and Theorem 8.2 of [9], we have

$$
\Theta\left(W_{u}\left(S_{k_{0}}^{\infty}\right)\right) \simeq \bigcup_{w_{p}^{\infty}<\Theta\left(W_{u}\left(S_{k_{0}}^{\infty}\right)\right)} W_{u}^{\infty}\left(w_{p}^{\infty}\right),
$$

where $\simeq$ denotes retracts by deformation.

Observe that $W_{u}\left(S_{k_{0}}^{\infty}\right)$ is a stratified set of dimension at most $k_{0}$, since each $W_{u}^{\infty}\left(w_{p}^{\infty}\right)$ is a manifold of dimension $i\left(w_{p}^{\infty}\right)$. Therefore, $\Theta\left(W_{u}\left(S_{k_{0}}^{\infty}\right)\right)$ is also a stratified set of top dimension $k_{0}+1$. By transversality arguments and by dimension's reason, the stable manifold of any critical point at infinity of Morse index larger than or equal to $k+2$ can be avoided during such a deformation. Therefore,

$$
\Theta\left(W_{u}\left(S_{k_{0}}^{\infty}\right)\right) \simeq \bigcup_{\substack{w_{p}^{\infty}<\Theta\left(W_{u}\left(S_{k_{0}}^{\infty}\right)\right) \\ i\left(w_{p}^{\infty}\right) \leq k_{0}+1}} W_{u}^{\infty}\left(w_{p}^{\infty}\right),
$$

which can be written as

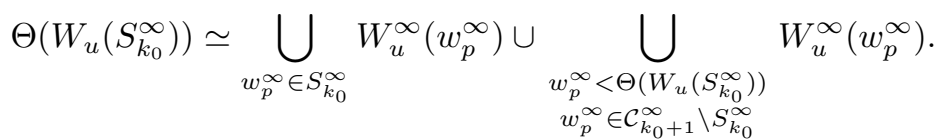

Working now by the contraction $\Theta_{0}$ provided by assumption $\left(\mathrm{A}_{1}\right)$ of Theorem 1.2 , there is no critical point at infinity in $\mathcal{C}_{k_{0}+1}^{\infty} \backslash S_{k_{0}}^{\infty}$ which is dominated by $\Theta_{0}\left(W_{u}\left(S_{k_{0}}^{\infty}\right)\right)$. Thus,

$$
\Theta_{0}\left(W_{u}\left(S_{k_{0}}^{\infty}\right)\right) \simeq \bigcup_{w_{p}^{\infty} \in S_{k_{0}}^{\infty}} W_{u}^{\infty}\left(w_{p}^{\infty}\right)
$$


Taking the Euler-Poincaré characteristic (denoted $\chi$ ) of both sides, we get

$$
\chi\left(\Theta_{0}\left(W_{u}\left(S_{k_{0}}^{\infty}\right)\right)\right)=1=\sum_{w_{p}^{\infty} \in S_{k_{0}}^{\infty}}(-1)^{i\left(w_{p}^{\infty}\right)},
$$

since $\Theta_{0}\left(W_{u}\left(S_{k_{0}}^{\infty}\right)\right)$ is a contractible set. This yields a contradiction with assumption $\left(\mathrm{A}_{2}\right)$ of Theorem 1.2. This concludes the proof of Theorem 1.2.

Proof of Corollary 1.3. Set $k_{0}:=\sup \left\{i\left(w_{p}^{\infty}\right), w_{p}^{\infty} \in \mathcal{C}^{\infty}\right\}$. For $S_{k_{0}}^{\infty}=$ $\mathcal{C}_{k_{0}}^{\infty}$, assumption $\left(\mathrm{A}_{1}\right)$ of Theorem 1.2 is satisfied for any contraction $\Theta\left(W_{u}\left(S_{k_{0}}^{\infty}\right)\right)$ in $\Sigma^{+}$, since $\mathcal{C}_{k_{0}+1}^{\infty} \backslash S_{k_{0}}^{\infty}=\emptyset$. In addition, assumption $\left(\mathrm{A}_{2}\right)$ of Theorem 1.2 is satisfied from the assumption of Corollary 1.3. Therefore Theorem 1.2 applies. $\square$

Proof of Corollary 1.4. Set $k_{0}:=\sup \left\{i\left(y_{\infty}\right), y \in \mathcal{K}^{+}\right\}$. For $S_{k_{0}}^{\infty}=$ $\left\{(y)_{\infty}, y \in \mathcal{K}^{+}\right\}$, assumption $\left(\mathrm{A}_{2}\right)$ of Theorem 1.2 is satisfied from the condition of Corollary 1.4. Thus, to achieve the proof, it remains to construct a contraction $\Theta_{0}\left(W_{u}\left(S_{k_{0}}^{\infty}\right)\right)$ satisfying assumption $\left(\mathrm{A}_{1}\right)$ of Theorem 1.2.

Since $H$ is of the form $H=1+\varepsilon H_{0}, H_{0} \in C^{2}\left(\mathbb{S}^{n-1}\right)$ and $|\varepsilon|$ is small, the functional is

$$
J_{\varepsilon}(u)=\frac{1}{\left(\int_{\mathbb{S}^{n-1}}\left(1+\varepsilon H_{0}\right) u^{2(n-1) /(n-2)}\right)^{(n-2) /(n-1)}} .
$$

In the case of the Yamabe functional $J_{0}$, the minimum of $J_{0}$, denoted by $S_{n}$, is achieved by the $n$-dimensional manifold $Z$ of critical points given by

$$
Z=\left\{\delta_{(a, \lambda)}, a \in \mathbb{S}^{n-1}, \lambda>0\right\} .
$$

For $\alpha \in \mathbb{R}$, we set $J_{\varepsilon}^{\alpha}:=\left\{u \in \Sigma^{+}, J_{\varepsilon}(u) \leq \alpha\right\}$. Since $J_{\varepsilon}(u)=J_{0}(u)(1+O(\varepsilon))$, with $O(\varepsilon)$ independent of $u$, we get for $|\varepsilon|$ small enough

$$
J_{\varepsilon}^{S_{n}+\eta} \subset J_{0}^{S_{n}+2 \eta} \subset J_{\varepsilon}^{S_{n}+3 \eta} .
$$

For $\eta=S_{n} / 4$ and for $|\varepsilon|$ sufficiently small, the critical points at infinity made of two bubbles or more are above the level $S_{n}+3 \eta$ and the ones with a single bubbles are below $S_{n}+\eta$. Thus, $J_{\varepsilon}^{S_{n}+3 \eta} \simeq J_{\varepsilon}^{S_{n}+\eta}$ and, therefore, $J_{0}^{S_{n}+2 \eta} \simeq J_{\varepsilon}^{S_{n}+\eta}$. Observe that $Z$ is contractible and it is a retract by deformation of $J_{0}^{S_{n}+2 \eta}$. We derive thus that $J_{\varepsilon}^{S_{n}+\eta}$ is a contractible set. Since $W_{u}\left(S_{k_{0}}^{\infty}\right)=\bigcup_{y \in \mathcal{K}^{+}} W_{u}^{\infty}(y)_{\infty}$ is in $J_{\varepsilon}^{S_{n}+\eta}$, then there exists at least one contraction $\Theta_{0}\left(W_{u}\left(S_{k_{0}}^{\infty}\right)\right)$ of $W_{u}\left(S_{k_{0}}^{\infty}\right)$ in $J_{\varepsilon}^{S_{n}+\eta}$. This contraction avoids the stable manifold of any critical point at infinity which is not in $S_{k_{0}}^{\infty}$. Hence assumption $\left(\mathrm{A}_{1}\right)$ of Theorem 1.2 follows.

Related existence results. Next we provide some existence results through prescribed functions $H$ which satisfy the assumptions of Theorem 1.2 independently of the assumptions of Corollaries 1.3 and 1.4.

Theorem 3.1. For $n=4$, if there exists $k_{0} \in \mathbb{N}$ such that 
$\left(\mathrm{C}_{1}\right) i\left(w_{p}^{\infty}\right) \neq k_{0}+1$, for all $w_{p}^{\infty} \in \mathcal{C}^{\infty}$,

$\left(\mathrm{C}_{2}\right) \sum_{w_{p}^{\infty} \in \mathcal{C}_{k_{0}}^{\infty}}(-1)^{i\left(w_{p}^{\infty}\right)} \neq 1$,

then (1.1) has a solution.

Proof. For $S_{k_{0}}^{\infty}=\mathcal{C}_{k_{0}}^{\infty}$, let $\Theta_{0}\left(W_{u}\left(S_{k_{0}}^{\infty}\right)\right)$ be a contraction of $W_{u}\left(S_{k_{0}}^{\infty}\right)$ in $\Sigma^{+}$. Observe that under assumption $\left(\mathbf{C}_{1}\right), \Theta_{0}\left(W_{u}\left(S_{k_{0}}^{\infty}\right)\right)$ satisfies assumption $\left(\mathrm{A}_{1}\right)$ of Theorem 1.2 and $\left(\mathrm{A}_{2}\right)$ follows from $\left(\mathrm{C}_{2}\right)$.

We point out that Theorem 3.1 extends Corollary 1.3 in dimension 4 to include the case where the total sum given in Corollary 1.3 equals 1 but a partial one is not provided that there is a jump in the Morse indices of elements of $\mathcal{C}^{\infty}$. Notice that Theorem 3.1 is not applicable in dimension $n \neq 4$, since it is exactly equivalent to the theorem of [3] and [16] in dimension 3 and for dimension $n \geq 5$, under $\left(\mathrm{C}_{1}\right), \sum_{w_{p}^{\infty} \in \mathcal{C}_{k_{0}}^{\infty}}(-1)^{i\left(w_{p}^{\infty}\right)}$ is always equals 1 .

ThEOREM 3.2. Let $n \geq 3$. Assume that the critical points of $H$ are ordered as follows $H\left(y_{0}\right) \geq H\left(y_{1}\right) \geq \ldots \geq H\left(y_{\ell}\right)$ with

$\left(\mathrm{H}_{0}\right) W_{s}\left(y_{i}\right) \cap W_{u}\left(y_{j}\right)=\emptyset$, for all $y_{i} \in \mathcal{K}^{+}, y_{j} \notin \mathcal{K}^{+}$.

Here $W_{s}(y)$ and $W_{u}(y)$ are the stable and the unstable manifold of the gradient vector field $(-\partial H)$ at $y$.

For every $j=0, \ldots, \ell$, we denote

$$
X_{j}=\bigcup_{\substack{y_{i} \in \mathcal{K}^{+} \\ 0 \leq i \leq j}} W_{s}\left(y_{i}\right) .
$$

Under the following assumptions:

$\left(\mathrm{H}_{1}\right)$ there exists $j_{0}, 0 \leq j_{0} \leq \ell$ such that $X_{j_{0}}$ is contractible in $H_{c_{0}}:=\{x \in$ $\left.\mathbb{S}^{n-1}, H(x) \geq c_{0}\right\}$, where

$$
H\left(y_{k}\right)<c_{0} \leq H\left(y_{j_{0}}\right), \quad \text { for all } k \geq j_{0}+1 \text {, with } y_{k} \in \mathcal{K}^{+},
$$

(H $\mathrm{H}_{2} \sum_{\substack{y_{i} \in \mathcal{K}^{+} \\ 0 \leq i \leq j_{0}}}(-1)^{n-1-\operatorname{ind}\left(H, y_{i}\right)} \neq 1$,

equation (1.1) has a solution provided $H$ is close to a constant function.

Proof. Setting $k_{0}=\sup \left\{i\left(y_{i}\right)_{\infty}, y_{i} \in \mathcal{K}^{+}, 0 \leq i \leq j_{0}\right\}$ and $S_{k_{0}}^{\infty}=$ $\left\{\left(y_{i}\right)_{\infty}, y_{i} \in \mathcal{K}^{+}, 0 \leq i \leq j_{0}\right\}$. Under assumption $\left(\mathrm{H}_{2}\right)$, assumption $\left(\mathrm{A}_{2}\right)$ of Theorem 1.2 holds. Now to construct a contraction $\Theta_{0}\left(W_{u}\left(S_{k_{0}}^{\infty}\right)\right)$ of $S_{k_{0}}^{\infty}$ in $\Sigma^{+}$ satisfying assumption $\left(\mathrm{A}_{1}\right)$ of Theorem 1.2 , we proceed as follows.

Recall that for every $y_{i} \in \mathcal{K}^{+}$the level of $J$ at the critical point at infinity $\left(y_{i}\right)_{\infty}$ is $c\left(y_{i}\right)_{\infty}:=S_{n} /\left(H\left(y_{i}\right)\right)^{(n-2) /(n-1)}$ and the unstable manifold at infinity 
$W_{u}^{\infty}\left(y_{i}\right)_{\infty}$ can be described under assumption $\left(\mathrm{H}_{0}\right)$ by $W_{s}\left(y_{i}\right) \times[A,+\infty[$, the domain of the variable $\lambda$, for a positive earl $A$ large enough. Thus,

$$
W_{u}\left(S_{k_{0}}^{\infty}\right) \equiv X_{j_{0}} \times\left[A,+\infty\left[\quad \text { by } \delta_{(x, \lambda)} \leftrightarrow(x, \lambda) .\right.\right.
$$

It follows from $\left(\mathrm{H}_{1}\right)$ that there exists

$$
\phi:[0,1] \times X_{j_{0}} \rightarrow H_{c_{0}}, \quad(t, x) \mapsto \phi(t, x),
$$

continuous such that, for all $x \in X_{j_{0}}, \phi(0, x)=x$ and $\phi(1, x)=a_{0}$, a fixed point of $H_{c_{0}}$. Such contraction gives rise to the following contraction:

$$
\Theta_{0}:[0,1] \times W_{u}\left(S_{k_{0}}^{\infty}\right) \rightarrow \Sigma^{+}, \quad\left(t, \delta_{(x, \lambda)}\right) \mapsto \delta_{(\phi(t, x), \lambda)} .
$$

For all $\delta_{(x, \lambda)} \in W_{u}\left(S_{k_{0}}^{\infty}\right), \Theta_{0}\left(0, \delta_{(x, \lambda)}\right)=\delta_{(x, \lambda)}$ and $\Theta_{0}\left(1, \delta_{(x, \lambda)}\right)=\delta_{\left(a_{0}, \lambda\right)}$, a fixed point in $\Sigma^{+}$. Expanding $J$ along this homotopy, we find

$$
\begin{aligned}
J\left(\delta_{(\phi(t, x), \lambda)}\right) & =\frac{S_{n}}{(H(\phi(t, x)))^{(n-2) /(n-1)}}\left(1+O\left(A^{-2}\right)\right) \\
& \leq \frac{S_{n}}{\left(c_{0}\right)^{(n-2) /(n-1)}}\left(1+O\left(A^{-2}\right)\right) .
\end{aligned}
$$

Let $c_{1}:=S_{n} /\left(c_{0}\right)^{(n-2) /(n-1)}$ and let $\varepsilon>0$ be very small so that $c_{1}+\varepsilon<c\left(y_{k}\right)_{\infty}$, for all $y_{k} \in \mathcal{K}^{+}$and $k \geq j_{0}+1$. Thus, for $A$ large enough, the contraction $\Theta_{0}\left(W_{u}\left(S_{k_{0}}^{\infty}\right)\right)$ is performed under the level $c_{1}+\varepsilon$. Using the fact that $H$ is close to constant, all critical points at infinity of two masses or more are above $c_{1}+\varepsilon$. Therefore, all critical points at infinity which are not in $S_{k_{0}}^{\infty}$ are above $c_{1}+\varepsilon$, so it cannot be dominated by $\Theta_{0}\left(W_{u}\left(S_{k_{0}}^{\infty}\right)\right)$. Hence condition $\left(\mathrm{A}_{1}\right)$ of Theorem 1.2 is satisfied for $\Theta_{0}\left(W_{u}\left(S_{k_{0}}^{\infty}\right)\right)$.

REmark 3.3. We can obtain $\left(\mathrm{H}_{0}\right),\left(\mathrm{H}_{1}\right)$ and $\left(\mathrm{H}_{2}\right)$ of the above theorem through the following situation. We order the critical points of $H$ as follows:

$$
H\left(y_{0}\right) \geq H\left(y_{1}\right)>H\left(y_{2}\right)>H\left(y_{3}\right) \geq \cdots \geq H\left(y_{\ell}\right) .
$$

(a) Assume that $y_{0}$ and $y_{1}$ are two maxima of $H$ on $\mathbb{S}^{n-1}$. Thus, $y_{0}, y_{1} \in \mathcal{K}^{+}$ and $\operatorname{ind}\left(H, y_{0}\right)=\operatorname{ind}\left(H, y_{1}\right)=n-1$.

(b) Assume that $y_{2} \notin \mathcal{K}^{+}$with $\operatorname{ind}\left(H, y_{2}\right)=n-2$. Thus $W_{s}\left(y_{2}\right)$ is a 1dimensional manifold.

(c) Assume that $W_{s}\left(y_{2}\right)$ is with boundary. Thus, the critical points $y_{0}$ and $y_{1}$ form the boundary of $W_{s}\left(y_{2}\right)$.

(d) Assume that $\mathcal{K}^{+}=\left\{y_{0}, y_{1}, y_{3}\right\}$ with $\operatorname{ind}\left(H, y_{3}\right)=n-2$.

Observe that condition $\left(\mathrm{H}_{0}\right)$ follows from (a)-(d) and the following dimension argument:

$$
W_{s}(z) \cap W_{u}\left(z^{\prime}\right) \neq \emptyset \Rightarrow \operatorname{ind}\left(H, z^{\prime}\right) \geq \operatorname{ind}(H, z)+1 .
$$

For condition $\left(\mathrm{H}_{1}\right)$, we take $j_{0}=1, X_{j_{0}}=W_{s}\left(y_{1}\right) \cup W_{s}\left(y_{0}\right)=\left\{y_{1}, y_{0}\right\}$. From (c), $X_{j_{0}}$ is contractible in $\overline{W_{s}\left(y_{2}\right)}$, the closer of $W_{s}\left(y_{2}\right)$. Therefore, $X_{j_{0}}$ is contractible in $H_{c_{0}}$ with $c_{0}=H\left(y_{2}\right)$. 
Lastly, condition $\left(\mathrm{H}_{2}\right)$ is satisfied since

$$
\sum_{\substack{y_{i} \in \mathcal{K}^{+} \\ 0 \leq i \leq 1}}(-1)^{n-1-\operatorname{ind}\left(H, y_{i}\right)}=2 .
$$

Hence, equation (1.1) has a solution even $\sum_{y \in \mathcal{K}^{+}}(-1)^{n-1-\operatorname{ind}(H, y)}=1$ in this situation.

\section{REFERENCES}

[1] W. Abdelhedi and H. Chtioui, The Prescribed boundary mean curvature problem on standard n-dimensional ball, Nonlinear Anal. 67 (2007), 668-686.

[2] W. Abdelhedi, H. Chtioui and M. Ould Ahmedou, A Morse theoretical approach for boundary mean Curvature Problem on $\mathbb{B}^{4}$, J. Funct. Anal. 254 (2008), 1307-1341.

[3] _ Conformal metrics with prescribed boundary mean curvature on balls, Ann. Global Anal. Geom. 36 (2009), Number 4, 327-362.

[4] M.A. Al-Ghamdi, H. Chtioui and K. Sharaf, Topological methods for boundary mean curvature problem on $B^{n}$, Adv. Nonlinear Stud. 14 (2) (2014), 445-461.

[5] T. Aubin, Some nonlinear problems in Riemannian geometry, Springer Monographs Math., Springer Verlag, Berlin 1998.

[6] A. BAHri, Critical point at infinity in some variational problems, Pitman Res. Notes Math. Ser. 182, Longman Sci. Tech. Harlow 1989.

[7] _ An invariant for yamabe-type flows with applications to scalar curvature problems in high dimensions, A celebration of J. F. Nash Jr., Duke Math. J. 81 (1996), 323-466. Notes Math. Ser. 182, Longman Sci. Tech. Harlow 1989.

[8] A. BAHRI AND J. M. CoRON, The scalar curvature problem on the standard three dimensional spheres, J. Funct. Anal. 95, (1991), 106-172.

[9] A. Bahri and P. Rabinowitz, Periodic orbits of hamiltonian systems of three body type, Ann. Inst. H. Poincaré Anal. Non Linéaire 8 (1991), 561-649.

[10] S.A. Chang, M.J. Gursky AND P.C. YANG, The scalar curvature equation on 2 and 3 spheres, Calc. Var. 1 (1993), 205-229.

[11] S.A. Chang, X. Xu And P. C. YAng, A perturbation result for prescribing mean curvature, Math. Ann 310 (3) (1998), 473-496.

[12] P. Cherrier, Problèmes de Neumann non linéaires sur les variétés Riemanniennes, J. Funct. Anal. 57 (1984), 154-207.

[13] Z. Djadli, A. Malchiodi and M. Ould Ahmedou, The prescribed boundary mean curvature problems on $B^{4}$, J. Differential Equations 206 (2004), 373-398.

[14] J.F. Escobar, Conformal deformation of Riemannian metric to scalar flat metric with constant mean curvature on the boundary, Ann. of Math. 136 (1992), 1-50.

[15] _ Conformal metric with prescribed mean curvature on the boundary, Calc. Var. Partial Differential Equations 4 (1996), 559-592.

[16] J.F. Escobar And G. Garcia, Conformal metric on the ball with zero scalare and prescribed mean curvature on the boundary, J. Funct. Anal. 211:1 (2004), 71-152.

[17] V. Felli and M. Ould Ahmedou, On a geometric equation with critical nonlinearity on the boundary, Pacific J. Math. 219 (2005), 1-25.

[18] Z.C. HAN AND Y.Y. LI, The Yamabe problem on manifolds with boundary existence and compactness results, Duke Math. J. 99, (1999), 489-542. 
[19] _ The existence of conformal metrics with constant scalar curvature and constant boundary mean curvature, Comm. Anal. Geom. 8, (2000), 809-869.

[20] J. KAZDAN AND F. WARNER, Existence and conformal deformation of metrics with prescribed Gaussian and scalar curvatures, Ann. of Math (2) 101 (1975), 317-331.

[21] Y.Y. Li AND M. ZHu, Uniqueness theorems through the method of moving spheres, Duke Math. J. 80 (1995), 383-417.

[22] K. Sharaf, H. Alharthy and S. Altharwi, Conformal transformation of metrics on the $n$-ball, Nonlinear Anal. 95 (2014), 246-262.

Manuscript received December 10, 2014

accepted March 24, 2015

KHADIJAH SHARAF

Department of Mathematics

King Abdulaziz University

P.O. 80230, Jeddah, KINGDOM OF SAUDI ARABIA

E-mail address: kh-sharaf@yahoo.com 